\title{
Friction coefficient for deep-inelastic heavy-ion collisions.
}

\author{
G.G.Adamian ${ }^{1,2}$, R.V.Jolos ${ }^{1}$ and A.K. Nasirov ${ }^{1,2}$ \\ ${ }^{1}$ Joint Institute for Nuclear Research, Dubna, 141980 Russia \\ A.I.Muminov \\ ${ }^{2}$ Heavy Ion Physics Department, Institute of Nuclear Physics 702132 Ulugbek, Tashkent, \\ Uzbekistan
}

(September 30, 2018)

\begin{abstract}
Based on the microscopic model, the friction coefficient for the relative motion of nuclei in deep-inelastic heavy-ion collisions is calculated. The radial dependence of the friction coefficient is studied and the results are compared with those found by other methods. Based on this result, it was demonstrated that the kinetic energy dissipation in deep-inelastic heavy-ion collisions is a gradual process which takes up a significant part of a reaction time. An advantage of the suggested method is that it allows one to consider the relative motion of nuclei and the intrinsic motion self-consistently.
\end{abstract}

25.70.Gh, 25.70.Jj

Typeset using REVTEX 


\section{INTRODUCTION}

Nuclear friction is an important ingredient of theoretical approaches to a variety of nuclear physics phenomena, such as dynamic thresholds for compound nucleus formation [15], enhancement of neutron emission prior to fission [6,7], width of mass and charge distributions in deep-inelastic heavy-ion reactions [8], and the width of giant resonances 9]. There are many experimental results on deep-inelastic heavy-ion collisions (DIC) and fusion-fission reactions which need the introduction of the nuclear friction concept for their interpretation. This stresses the importance of understanding the nature of nuclear friction.

The present paper is devoted to calculations of the friction coefficient for DIC heavy-ion collisions. Its appearance is stimulated not only by the possibility to perform more exact calculations than earlier, but also by the new experimental results which require a more detailed microscopic theory for their interpretation than was necessary before.

Different theoretical approaches to this problem are known. The majority of them are based on the assumption that the dissipative mechanism is of a one-body nature [10 12]. These models differ in the structure of the intrinsic excitations that are taken into account. These can be pure collective excited states 13 16 or incoherent particle-hole excitations [10,18, 19. Some of the models do not include nucleon exchange and consider only particlehole excitations, with both the particle and the hole belonging to the same nucleus [20] or vice versa [10]. The models also differ in the approximations they use for including the finite decay time of one particle-one hole $(1 \mathrm{p}-1 \mathrm{~h})$ excitations in more complicated configurations (2p-2h, and so on). Many approaches [12, 13, 18,21] implicitly use the statistical assumption of rapid equilibration of the noncollective intrinsic degrees of freedom and, therefore are not applicable to the description of the initial phase of the reactions, where the main part of kinetic energy dissipation takes place.

The contribution of the actual $1 \mathrm{p}-1 \mathrm{~h}$ state or more complicated ones to the dissipation process depends on the occupation numbers of the single-particle states and their evolution during the reaction. However, in the calculations of the nuclear friction coefficient performed 
up to now, the statistical assumption on the excitation energy distribution was realized in the usual way, meaning the introduction of temperature and, correspondingly, of the Fermi occupation numbers at the very beginning of the reaction. In principle, temperature introduced in this way is a time-dependent quantity. However, in practice temperature was considered to be a constant corresponding to the total excitation energy [15, 17]. Thus, the time dependence of the single-particle occupation numbers was not taken into account. Only in the approach based on the Dissipative Diabatic Dynamics (DDD) 22, 23] was the evolution of the single-particle occupation numbers taken into account, but under the assumption of diabaticity. There are some doubts however, about the validity of the DDD concept. It is also known from the calculations of inelastic processes in nucleus-nucleus collisions that appreciable energy dissipation takes place even before the first crossing of the single-particle levels near the Fermi surface [24].

We should also mention the approach suggested in 25], where relative and intrinsic motion were consistently treated in a time-dependent theory of heavy-ion collisions. The authors presumed neither weak coupling between the relative motion and the intrinsic excitations nor the canonical distribution function for the density operator of intrinsic motion. However, the analytical expressions for the friction tensor and other characteristics of the energy transport obtained in 25] were not applied to calculate them.

Thus, it is the aim of the present paper to take into account the time evolution of the single-particle occupation numbers during the reaction by numerical solution of the master equation for them and, based on this result, to perform calculations for the friction coefficient. Since the occupation numbers found in this way correspond to the current kinetic energy losses, this means that the relative and intrinsic motions are considered selfconsistently. Our model makes it possible to take into account explicitly the influence of the nuclear shell structure on the collision process. Moreover, we improved the single-particle approximation by a phenomenological allowance for the residual interaction which is treated in the so called $\tau$-approximation. The radial friction coefficient is calculated as a function of the mass and charge of the reaction participants. 
The general formalism is given in Section 2. The results of the calculations are presented in Section 3. A summary is given in Section 4.

\section{BASIC FORMALISM}

It is convenient to start with the total Hamiltonian of a dinuclear system written in the form

$$
\hat{H}=\hat{H}_{r e l}(\mathbf{R} ; \mathbf{P})+\hat{H}_{i n}(\xi)+\delta \hat{V}(\mathbf{R}, \xi),
$$

where the Hamiltonian of a relative motion,

$$
\hat{H}_{r e l}(\mathbf{R} ; \mathbf{P})=\frac{\hat{\mathbf{P}}^{2}}{2 \mu}+\hat{\mathcal{V}}(\hat{\mathbf{R}}),
$$

consists of the kinetic energy operator and the nucleus-nucleus interaction potential $\hat{\mathcal{V}}(\hat{\mathbf{R}})$.

Here, $\hat{\mathbf{R}}$ is the relative distance between the centers of mass of the fragments, $\hat{\mathbf{P}}$ is the conjugate momentum, and $\mu$ is the reduced mass of the system; $\xi$ is a set of relevant

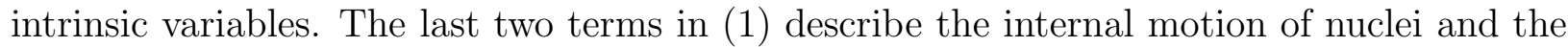
coupling between the relative and internal motions (for details, see [26,27]). It is clear that the coupling term leads to a dissipation of the kinetic energy into the energy of internal nucleon motion.

Neglecting at the moment the residual nucleon-nucleon interaction, whose effect will be included later, we take a sum of the last two terms (11) as a single-particle Hamiltonian of a dinuclear system

$$
\begin{aligned}
\hat{H}_{i n}(\xi)+\delta \hat{V}(\mathbf{R}, \xi) & =\hat{\mathcal{H}}(\mathbf{R}(t), \xi)+h_{\text {residual }}, \\
\hat{\mathcal{H}}(\mathbf{R}(t)) & =\sum_{i=1}^{A}\left(\frac{-\hbar^{2}}{2 m} \Delta_{i}+\hat{V}_{P}\left(\mathbf{r}_{i}-\mathbf{R}(t)\right)+\hat{V}_{T}\left(\mathbf{r}_{i}\right)\right),
\end{aligned}
$$

where $m$ is the nucleon mass and $A=A_{P}+A_{T}$ is the total number of nucleons in the system.

Then, in the second quantization representation, the Hamiltonian $\hat{\mathcal{H}}(\mathbf{R}(t), \xi)$ can be written as 


$$
\hat{\mathcal{H}}(\mathbf{R}(t), \xi)=\sum_{P} \varepsilon_{P} \mathrm{a}_{P}^{+} \mathrm{a}_{P}+\sum_{T} \varepsilon_{T} \mathrm{a}_{T}^{+} \mathrm{a}_{T}+\sum_{i \neq i^{\prime}} V_{i i^{\prime}}(\mathbf{R}(t)) \mathrm{a}_{i}^{+} \mathrm{a}_{i^{\prime}}
$$

where

$$
\begin{aligned}
& \sum_{i \neq i^{\prime}} V_{i i^{\prime}}(\mathbf{R}(t)) \mathrm{a}_{i}^{+} \mathrm{a}_{i^{\prime}}=\sum_{P \neq P^{\prime}} \Lambda_{P P^{\prime}}^{(T)}(\mathbf{R}(t)) \mathrm{a}_{P}^{+} \mathrm{a}_{P^{\prime}}+\sum_{T \neq T^{\prime}} \Lambda_{T T^{\prime}}^{(P)}(\mathbf{R}(t)) \mathrm{a}_{T}^{+} \mathrm{a}_{T^{\prime}}+ \\
& \sum_{T, P} \mathrm{~g}_{P T}(\mathbf{R}(t))\left(\mathrm{a}_{P}^{+} \mathrm{a}_{T}+\text { h.c. }\right) .
\end{aligned}
$$

Here $P \equiv\left(n_{P}, j_{P}, l_{P}, m_{P}\right)$ and $T \equiv\left(n_{T}, j_{T}, l_{T}, m_{T}\right)$ are the sets of quantum numbers characterizing the single-particle state in an isolated projectile and the target nuclei, respectively. The single-particle basis is constructed from the asymptotic wave vectors of the singleparticle states of the noninteracting nuclei-the projectile ion $\mid P>$ and the target nucleus $\mid T>$ in the form

$$
\begin{aligned}
& |\tilde{P}>=| P>-\frac{1}{2} \sum_{T}|T><T| P> \\
& |\tilde{T}>=| T>-\frac{1}{2} \sum_{P}|P><P| T>.
\end{aligned}
$$

For this basis set, the orthogonality condition is satisfied up to terms linear in $\langle P \mid T\rangle$. Then

$$
\begin{array}{r}
\Lambda_{P P^{\prime}}^{(T)}(\mathbf{R}(t))=<P\left|V_{T}(\mathbf{r})\right| P^{\prime}> \\
\Lambda_{T T^{\prime}}^{(P)}(\mathbf{R}(t))=<T\left|V_{P}(\mathbf{r}-\mathbf{R}(t))\right| T^{\prime}>, \\
g_{P T}(\mathbf{R}(t))=\frac{1}{2}<P\left|V_{P}(\mathbf{r}-\mathbf{R}(t))+V_{T}(\mathbf{r})\right| T>.
\end{array}
$$

The nondiagonal matrix elements $\Lambda_{P P^{\prime}}^{(T)}\left(\Lambda_{T T^{\prime}}^{(P)}\right)$ generate the particle-hole transitions in the projectile (target) nucleus. The matrix elements $g_{P T}$ are responsible for the nucleon exchange between reaction partners. These matrix elements were calculated using the approach proposed in 28,29]. In (四), $\varepsilon_{P(T)}$ are the single-particle energies of nonperturbed states in the projectile (target) nucleus. The coupling between the intrinsic nuclear degrees of freedom and the collective variable $\mathbf{R}$ is introduced by the $\mathbf{R}$ dependence of the sum of the singleparticle potentials in (3). Since the trajectory calculation shows that the relative distance $\mathbf{R}(t)$ between the centers of the interacting nuclei could not be less than the sum of their 
radii the tail of the partner single-particle potentials can be considered as a perturbation disturbing the asymptotic single-particle wave functions and their energies.

It is convenient to include the diagonal matrix elements of $V_{i i^{\prime}}(\mathbf{R}(t))$ in $H_{\text {in }}$, introducing the renormalized $\mathbf{R}(t)$-dependent single-particle energies

$$
\begin{array}{r}
\tilde{\varepsilon}_{P}(\mathbf{R}(t))=\varepsilon_{P}+<P\left|V_{T}(\mathbf{r})\right| P>, \\
\tilde{\varepsilon}_{T}(\mathbf{R}(t))=\varepsilon_{T}+<T\left|V_{P}(\mathbf{r}-\mathbf{R}(t))\right| T>.
\end{array}
$$

When the nuclear forces begin to act between the colliding nuclei, the velocity of their relative motion can be considered as a small quantity compared to the Fermi velocity. Then the speed of the nucleons is mainly associated with their intrinsic motion. Since the relative (collective) motion is rather slow compared to the intrinsic motion, the perturbation of the intrinsic motion produced by changing the coupling to the relative motion $(\overline{\mathbf{R}})$ can be assumed to be small during some small time interval $\Delta t$ of an arbitrarily chosen time $t$ [13]. The small parameter in our consideration $\Delta t$ thus characterizes the time interval during which the $\overline{\mathbf{R}}$-dependent mean field of the combined dinuclear system changes so little that we can neglect the effect of this changing on the intrinsic motion. At the same time, the characteristic time $\Delta t$ can not be taken smaller than the relaxation time of the mean field. The situation described above is suitable for applying the linear response theory to a description of dissipative heavy-ion collisions [13]. For this reason, we start from the expression for the friction coefficient of the radial motion obtained in that approach [13],

$$
\begin{gathered}
\gamma_{R R}(R(t))=\sum_{i k}\left|\frac{\partial V_{i k}(\mathbf{R})}{\partial R}\right|^{2} B_{i k}^{(1)}(t) . \\
B_{i k}^{(n)}(t)=\frac{2}{\hbar} \int_{t-\Delta t}^{t} d t^{\prime} \frac{\left(t^{\prime}-t\right)^{n}}{n !} \exp \left(\frac{t^{\prime}-t}{\tau_{i k}}\right) \sin \left[\tilde{\omega}_{k i}\left(\mathbf{R}\left(t^{\prime}\right)\right)\left(t-t^{\prime}\right)\right] \\
\times\left[\tilde{n}_{k}\left(t^{\prime}\right)-\tilde{n}_{i}\left(t^{\prime}\right)\right],
\end{gathered}
$$

where $\tau_{i j}=\tau_{i} \tau_{k} /\left(\tau_{i}+\tau_{k}\right) ; \tau_{i}$ is the parameter describing the damping of the single-particle motion. The expression for $\tau_{i}$ is derived in the theory of quantum liquids [30,31] (see Appendix A); $\hbar \omega_{i j}=\tilde{\varepsilon}_{i}(\overline{\mathbf{R}})-\tilde{\varepsilon}_{j}(\overline{\mathbf{R}})$ is the energy of the single-particle transition in one of 
the nuclei as well as between the interacting nuclei. The important ingredients of this formula are the occupation numbers of the single-particle states $\tilde{n}_{i}(t)$. Since the excitation energy of the interacting nuclei changes significantly during the course of the collision, it is necessary to take into account the time dependence of the occupation numbers. The importance of this point was already stressed in [13]. At the same time, new experimental data indicate that the assumption of the fast statistical equilibration of the excitation energy during the collision time, i.e., an introduction of a time-dependent temperature and Fermi occupation numbers is not adequate for the physical picture. As already mentioned in the Introduction, the calculations of $\gamma_{R R}$ performed up to now have been done under the assumption that the occupation numbers can be taken as the Fermi occupation numbers

$$
n_{j}=\left(1+\exp \left(\left(E_{j}-\lambda\right) / \Theta\right)\right)^{-1}
$$

where $\Theta$ is the temperature corresponding to the total excitation energy of a dinuclear system.

To find the time-dependent occupation numbers $n_{j}(t)$, we developed in [26,27, 32] a method which is described briefly below for completeness of the presentation.

Since explicit allowance for the residual interaction requires extensive calculations, it is customary to take the two-particle collision integral into account in linearized form $(\tau$ approximation).

Then, the equation for the single-particle density matrix $\tilde{n}$ takes the form

$$
i \hbar \frac{\partial \hat{\tilde{n}}(t)}{\partial t}=[\hat{\mathcal{H}}(\mathbf{R}(t)), \hat{\tilde{n}}(t)]-\frac{i \hbar}{\tau}\left[\hat{\tilde{n}}(t)-\hat{\tilde{n}}^{e q}(\mathbf{R}(t))\right]
$$

where $\tilde{n}^{e q}(\mathbf{R}(t))$ is a local quasi-equilibrium distribution, i.e. a Fermi distribution with the temperature $T(t)$ corresponding to the excitation energy at the internuclear distance $\overline{\mathbf{R}}(t)$. Substituting our Hamiltonian (4) into (11, we have

$$
\begin{aligned}
i \hbar \frac{\partial \hat{\tilde{n}}_{i}(t)}{\partial t} & =\sum_{k}\left[V_{i k}(\overline{\mathbf{R}}(t)) \tilde{n}_{k i}(t)-V_{k i}(\overline{\mathbf{R}}(t)) \tilde{n}_{i k}(t)\right] \\
& -\frac{i \hbar}{\tau_{i}}\left[\tilde{n}_{i}(t)-\tilde{n}_{i}^{e q}(t)\right]
\end{aligned}
$$


where $\tilde{n}_{i}$ is a diagonal matrix element of the density matrix. The approximate equation for nondiagonal matrix elements takes the form

$$
\begin{aligned}
i \hbar \frac{\partial \hat{\tilde{n}}_{i k}(t)}{\partial t} & =\hbar\left[\tilde{\omega}_{i k}(\overline{\mathbf{R}}(t))-\frac{2 i}{\tau_{i k}}\right] \tilde{n}_{i k}(t) \\
& +V_{k i}(\overline{\mathbf{R}}(t))\left[\tilde{n}_{k}(t)-\tilde{n}_{i}(t)\right]
\end{aligned}
$$

where we have used the notations $\tilde{\omega}_{i k}=\left[\tilde{\varepsilon}_{i}-\tilde{\varepsilon}_{k}\right] / \hbar$.

Assuming incoherence in the phases of the nondiagonal matrix elements, we use the following approximation to simplify equation (13):

$$
\sum_{k^{\prime}} V_{k^{\prime} i}(\overline{\mathbf{R}}(t)) n_{k^{\prime} k}(t)-\sum_{i^{\prime}} V_{k i^{\prime}} n_{i i^{\prime}}(t) \approx V_{k i}(\overline{\mathbf{R}}(t))\left[n_{k}(t)-n_{i}(t)\right]
$$

As formulated above, we shall consider the solution of equations (12) and (13) for a small time interval $\Delta t$ of an arbitrarily choosen time $t$. Then the solution of equation (13) can be written as

$$
\begin{aligned}
\tilde{n}_{i k}(\bar{t}) & =\frac{1}{i \hbar} \int_{t}^{\bar{t}} d t^{\prime} V_{i k}\left(\mathbf{R}\left(t^{\prime}\right)\right) \exp \left\{i \int_{t^{\prime}}^{\bar{t}} d t^{\prime \prime}\left[\tilde{\omega}_{k i}\left(\mathbf{R}\left(t^{\prime \prime}\right)+\frac{i}{\tau_{i k}}\right]\right\}\right. \\
& \times\left[\tilde{n}_{k}\left(t^{\prime}\right)-\tilde{n}_{i}\left(t^{\prime}\right)\right],
\end{aligned}
$$

where $t \leq \bar{t} \leq t+\Delta t$. Substituting this result into Eq. (12) and transforming this equation to an integral, we obtain

$$
\begin{gathered}
\tilde{n}_{i}(\bar{t})=\exp \left(\frac{t-\bar{t}}{\tau_{i}}\right)\left\{\tilde{n}_{i}(t)+\frac{1}{\tau_{i}} \int_{t}^{\bar{t}} d t^{\prime} \tilde{n}_{i}^{e q}\left(\mathbf{R}\left(t^{\prime}\right)\right) \exp \left(\frac{t^{\prime}-t}{\tau_{i}}\right)\right. \\
\left.+\sum_{k} \int_{t}^{\bar{t}} d t^{\prime} \int_{t}^{t^{\prime}} d t^{\prime \prime} \Omega_{i k}\left(t^{\prime}, t^{\prime \prime}\right) \exp \left(\frac{t^{\prime \prime}-\bar{t}}{\tau_{i k}}\right)\left[\tilde{n}_{k}\left(t^{\prime \prime}\right)-\tilde{n}_{i}\left(t^{\prime \prime}\right)\right]\right\}
\end{gathered}
$$

where

$$
\Omega_{i k}\left(t, t^{\prime}\right)=\frac{2}{\hbar^{2}} \operatorname{Re}\left\{V_{i k}(\mathbf{R}(t)) V_{k i}\left(\mathbf{R}\left(t^{\prime}\right)\right) \exp \left[i \int_{t^{\prime}}^{t} d t^{\prime \prime} \tilde{\omega}_{k i}\left(\mathbf{R}\left(t^{\prime \prime}\right)\right)\right]\right\} .
$$

The formal solution of Eq. (15) is

$$
\tilde{n}_{i}(t)=\tilde{n}_{i}^{e q}(\mathbf{R}(t))\left[1-\exp \left(\frac{-\Delta t}{\tau_{i}}\right)\right]+n_{i}(t) \exp \left(\frac{-\Delta t}{\tau_{i}}\right),
$$


where

$$
n_{i}(\bar{t})=\tilde{n}_{i}(t)+\sum_{k} \int_{t}^{\bar{t}} d t^{\prime} \Omega_{i k}\left(t^{\prime}, t^{\prime}\right) \frac{\sin \left[\tilde{\omega}_{k i}\left(\mathbf{R}\left(t^{\prime}\right)\right)\left(t^{\prime}-t\right)\right]}{\tilde{\omega}_{k i}\left(\mathbf{R}\left(t^{\prime}\right)\right)}\left[\tilde{n}_{k}\left(t^{\prime}\right)-\tilde{n}_{i}\left(t^{\prime}\right)\right] .
$$

In fact, Eqs. (17) and (18) present an integral equation for $\tilde{n}_{i}(t)$.

\section{RESULTS AND DISCUSSION}

In this section, we present the results of calculating the radial friction coefficient and the kinetic energy losses as a function of the inter-nucleus distance for trajectories corresponding to DIC. The initial projectile energy, atomic masses, and charges of the colliding nuclei are the initial information used in the calculations. The single-particle potentials of the colliding nuclei are taken in the Woods-Saxon form with the parameters $r_{0}=1.18 \mathrm{fm}$ and $a=0.54$ fm. The characteristic time parameter $\Delta t$ is taken to be equal to $(0.8-1.0) \cdot 10^{-22} \mathrm{~s}$.

The equations of motion for $\mathbf{R}(t)$ and the single-particle occupation numbers $\tilde{n}_{i}(t)$ have been solved numerically, transforming differential equations into finite difference equations with the time step $\Delta t$ and the initial conditions $R(0)=20 \mathrm{fm}$ and $\tilde{n}_{i}(0)=1$ or 0 for occupied and unoccupied states of the noninteracting nuclei, respectively. Matrix elements $\Lambda_{P P^{\prime}}^{(T)}, \quad\left(\Lambda_{T T^{\prime}}^{(P)}\right)$, and $g_{P T}$ have been calculated using the procedure developed in 26,27.

The relative motion along the trajectory depends on the nucleus-nucleus interaction potential, which is determined by a double folding of the effective nuclear and Coulomb interactions of the nucleons with the nuclear densities of the interacting nuclei. Because of nucleon exchange and particle-hole excitations, the nuclear densities of the colliding nuclei evolve during the reaction and the nucleus-nucleus potential changes correspondingly. This effect is included in our calculations. As an example of these calculations we present the radial friction coefficient for the ${ }^{64} \mathrm{Zn}(440 \mathrm{MeV})+{ }^{196} \mathrm{Pt}$ reaction as a function of $R$ for an approach phase of the reaction (solid curve) in Fig. 1. The results of the classical model of D.H.E. Gross and Kalinowski [33] (dashed curves with stars) are shown for comparison, along with the results of the microscopic model developed in [15] which are obtained with 
a constant temperature (dashed curve without stars). The curves correspond to different temperatures which increase from $0.5 \mathrm{MeV}$ (bottom curve) to $2 \mathrm{MeV}$ (upper curve). It can be seen that the difference between our results and the results of [33] increases with the increase in the overlap of the colliding nuclei. Comparing our results with the results of [15], we can see that our radial friction coefficient coincides with those found in [15] for temperatures increasing as $R$ decreases. This kind of behavour of the radial friction coefficient obtained in [17]. It is natural since it corresponds to an increase in the kinetic energy loss during the approach phase of the reaction. Unfortunately, the results for larger values of $R$ are not presented in 15. Thus, our calculations demonstrate the importance of inclusion of the time dependence of the single-particle occupation numbers in the calculations.

In Figs.2 and 3, the radial friction coefficients $\gamma_{R R}$ are given as a function of $R$ for both approach (solid curve) and departure (dashed curve) stages of the ${ }^{64} \mathrm{Zn}(440 \mathrm{MeV})+{ }^{196} \mathrm{Pt}$ and ${ }^{56} \mathrm{Fe}(480 \mathrm{MeV})+{ }^{208} \mathrm{~Pb}$ reactions. It can be seen that $\gamma_{R R}$ takes a larger value for the approach stage compared to the departure stage. For the second reaction, this difference is significant.

In Fig. 4 and 5, the dependence of the total kinetic energy losses as calculated by our method (solid curve) and as based on the classical model of [33] realized with the code TRAJEC [34] is demonstrated for the ${ }^{64} \mathrm{Zn}(440 \mathrm{MeV})+{ }^{196} \mathrm{Pt}$ and ${ }^{56} \mathrm{Fe}(480 \mathrm{MeV})+{ }^{208} \mathrm{~Pb}$ reactions, respectively. The solid and dashed arrows indicate the moment corresponding to the turning point of the trajectory in our calculations and in the classical model, respectively. It can be seen that, in contrast to the results of the classical model, where the kinetic energy is dissipated during a short time interval of the order of $0.4 \cdot 10^{-21} \mathrm{~s}$ at the very beginning of the reaction, this process takes significantly larger time in our model. This result is a consequence of the smaller value of our friction coefficient relative to those used in the classical phenomenological models. As seen in Fig.1, the friction coefficient obtained in [33] significantly exceeds our friction coefficient where the interacting nuclei strongly overlap. Nevertheless, our friction coefficient reproduces the total amount of kinetic energy loss observed experimentally. If the results of the classical model calculations support the 
idea of the fast kinetic energy loss and thermalization, our calculations support the idea of gradual kinetic energy dissipation as was argued in [3, 35].

In Fig. 6, we show a correction to the nucleus-nucleus interaction potential connected with the rearrangement of nuclear densities during the approach phase of the reaction for the

collision of ${ }^{64} \mathrm{Ni}(320 \mathrm{MeV})+{ }^{208} \mathrm{~Pb}$. It can be seen that the correction increases in absolute value with a decrease of the internucleus distance $R$. For the same reaction, the nucleusnucleus interaction potential found in the sudden approximation (dotted line) is shown in Fig. 7 with the dynamical correction (dashed line), which is discussed just above, and the kinetic energy as a function of the trajectory (solid line). We show that the correction to the sudden approximation produced by a rearrangement of the particle distribution in the interacting nuclei is important and must be included in the consideration.

\section{SUMMARY}

In conclusion, we have calculated the friction coefficient for DIC based on the microscopic model of the structure of the colliding nuclei and avoiding the assumption of a fast statistical equilibrium of the dissipated kinetic energy. Our results demonstrate the importance of considering the friction coefficient as an exact dynamic function of the single-particle occupation numbers without the assumption of the fast statistical equilibrium of the excitation energy. Based on this result, we have demonstrated that the kinetic energy dissipation in DIC is a gradual process which takes up a significant part of a reaction time.

\section{ACKNOWLEDGMENTS}

We are grateful to Dr. H. Hofmann for the valuable discussions and suggestions which stimulated the writing of this paper, and we also want to thank Dr. N.V. Antonenko for fruitful discussions. We thank Dr. Zh. Kurmanov and Ms. Ann Schaeffer for their assistance in preparing this manuscript. The authors (G.G.A., R.V.J. and A.K.N.) are grateful to the International Science Foundation (Grant No. RFJ-000) and the Russian Foundation 
of Basic Research (Grant No. 95-02-05684) for financial support.

\section{APPENDIX A:}

The value of $\tau_{i}$ is calculated using the results of the theory of quantum liquids [30, 31]

$$
\begin{aligned}
\frac{1}{\tau_{i}^{(\alpha)}} & =\frac{\sqrt{2} \pi}{32 \hbar \varepsilon_{F_{K}}^{(\alpha)}}\left[\left(f_{K}-g\right)^{2}+\frac{1}{2}\left(f_{K}+g\right)^{2}\right]\left[\left(\pi \Theta_{K}\right)^{2}+\left(\tilde{\varepsilon}_{i}-\lambda_{K}^{(\alpha)}\right)^{2}\right] \\
& \times\left[1+\exp \left(\frac{\lambda_{K}^{(\alpha)}-\tilde{\varepsilon}_{i}}{\Theta_{K}}\right)\right]^{-1},
\end{aligned}
$$

where

$$
\Theta_{K}(t)=3.46 \sqrt{\frac{E_{K}^{*}(t)}{<A_{K}(t)>}}
$$

is the effective temperature determined by the amount of intrinsic excitation energy $E_{K}^{*}=$

$E_{K}^{*(Z)}+E_{K}^{*(N)} ;<A_{K}(t)>=<Z_{K}(t)>+<N_{K}(t)>, \lambda_{K}^{(\alpha)}(t)$, and $E_{K}^{*(\alpha)}(t)$ are the mass number, chemical potential, and intrinsic excitation energies for the proton $(\alpha=Z)$ and neutron $(\alpha=N)$ subsystems of the nucleus $K(K=P, T)$, respectively, (for details, see [26]). Furthermore, the finite size of nuclei and the available difference between the numbers of neutrons and protons need to use the following expressions for the Fermi energies [31]:

$$
\begin{aligned}
\varepsilon_{F_{K}}^{(Z)} & =\varepsilon_{F}\left[1-\frac{2}{3}\left(1+2 f^{\prime}\right) \frac{<N_{K}>-<Z_{K}>}{<A_{K}>}\right], \\
\varepsilon_{F_{K}}^{(N)} & =\varepsilon_{F}\left[1+\frac{2}{3}\left(1+2 f^{\prime}\right) \frac{<N_{K}>-<Z_{K}>}{<A_{K}>}\right],
\end{aligned}
$$

where $\epsilon_{F}=37 \mathrm{MeV}$,

$$
\begin{gathered}
f_{K}=f_{i n}-\frac{2}{<A_{K}>^{1 / 3}}\left(f_{i n}-f_{e x}\right), \\
f_{K}^{\prime}=f_{i n}^{\prime}-\frac{2}{<A_{K}>^{1 / 3}}\left(f_{i n}^{\prime}-f_{e x}^{\prime}\right)
\end{gathered}
$$

and $f_{i n}=0.09, f_{i n}^{\prime}=0.42, f_{e x}=-2.59, f_{e x}^{\prime}=0.54, g=0.7$ are the constants of the effective nucleonnucleon interaction. 


\section{REFERENCES}

[1] J.O. Newton, Sov. J. Part. Nucl. 21, 349 (1990).

[2] D.J. Hinde, Nucl. Phys. A553, 255c (1993).

[3] M. Thoenessen, E. Ramakrishnan, J.P. Beene, F.F. Bertrand, M.L. Halbert, D.J. Horen, P.E. Mueller, and R.L. Varner, Phys. Rev. C 51, 3148 (1995).

[4] J.L. Baretto, N.G. Nicolis, D.G. Sarantites, R.J. Charity, L.G. Sobotka, D.W. Stracener, D.C. Hensley, J.R. Beene, C. Baktash, M.L. Halbert, M. Thoennessen, Phys. Rev. C 51, $2584(1995)$.

[5] D.J. Hofmann, B.B. Back, and P.Paul, Phys. Rev. C 51, 2597 (1995).

[6] D. Hilsher and H. Rossner, Ann. Phys. (Paris) 17, 471 (1992).

[7] I.I. Gontchar, Sov. J. Part. Nucl., 26, 922 (1995).

[8] W.U. Schröder and J.R. Huizenga, In: Treatise on Heavy-Ion Science, D.A. Bromley (ed.) (Plenum Press, New York) Vol.2, p. 115 (1984).

[9] J.R. Nix and A.J. Sierk, Preprint LA-UR-87-133, Los Alamos National Laboratory, 1987.

[10] J. Randrup, Nucl. Phys. A307, 319 (1978); A327, 490 (1979).

[11] H. Feldmeier, Rep. Prog. Phys. 50, 1 (1987).

[12] J. Blocki, Y. Boneh, J.R. Nix, J. Randrup, M. Robel, A.J. Sierk, and W.J. Swiatecki, Ann. Phys. 112, 356 (1978); 113, 330 (1978); 125, 193 (1980).

[13] H. Hofmann and P.J. Siemens, Nucl. Phys. A257, 165 (1976); A275, 464 (1977); A288, $152(1977)$.

[14] A. Iwamoto, K. Harada, S. Yamaji, and S.Yoshida, Z. Phys. A302, 149 (1981).

[15] S. Yamaji and A. Iwamoto, Z. Phys. A313, 161 (1983).

[16] H. Hofmann, F.A. Ivanyuk, and S. Yamaji, Nucl. Phys. A598, 187 (1996); Preprint RIKEN-AF-NP-224, 2-1 Hirosawa, Wako, Saitama 351-01, Japan (1996).

[17] F.A. Ivanyuk, H. Hofmann, and S. Yamaji, Preprint RIKEN-AF-NP-232, 2-1 Hirosawa, Wako, Saitama 351-01, Japan (1996).

[18] H.A. Weidenmüller: Prog. Nucl. Part. Phys. 3, 49 (1980). 
[19] B.R. Barrett, S. Shlomo, H.A. Weidenmüller, Phys. Rev. C17, 17 (1978).

[20] R.V. Jolos, R. Schmidt, J. Teichert, Nucl. Phys. A249, 139 (1984).

[21] A. Gobbi and W. Nörenberg: In: Heavy-Ion Collisions. Bock R. (ed.), Amsterdam: North-Holland, Vol.II. (1980).

[22] A. Lukasiak, W. Cassing and W. Nörenberg, Nucl. Phys. A426, 181 (1984).

[23] D. Berdichevsky, A. Lukasiak, W. Nörenberg, and P. Rozmej, Nucl. Phys. A499, 609 (1989).

[24] H. J. Krappe, Nucl. Phys. A505, 417 (1989).

[25] N. Takigawa, K. Niita, Y. Okhuhara, and Yoshida Y., Nucl.Phys. A371, 130 (1981).

[26] G.G. Adamian, R.V. Jolos, and A.K. Nasirov, Z. Phys. A347, 203 (1994).

[27] G.G. Adamian, N.V. Antonenko, R.V. Jolos, and A.K. Nasirov, Phys. Part. Nucl. A25, $583(1994)$.

[28] G.G. Adamian, R.V. Jolos, and A.K. Nasirov, Sov. J. Nucl. Phys. 55, 660 (1992).

[29] G.G. Adamian, N.V. Antonenko, R.V. Jolos, and A.K. Nasirov, Nucl. Phys. A551, 321 (1993).

[30] D. Pines and P. Noziéres, Theory of Quantum Liquids, Benjamin, New York (1966).

[31] A.B. Migdal, Theory of the Finite Fermi-Systems and Properties of Atomic Nuclei, Moscow, Nauka (1983).

[32] G.G. Adamian, R.V. Jolos, A.I. Muminov, and A.K. Nasirov, Phys. Rev. C53, 871 (1996).

[33] D.H.E. Gross and H. Kalinowski, Phys. Reports 45, 175 (1978).

[34] R. Schmidt, Part. \& Nucl. 13, 1203 (1982).

[35] W.U. Schröder, J.R. Birkelund, J.R. Huizenga, K.L. Wolf, and V.E. Viola Jr., Phys. Rep. 45, 301 (1987). 


\section{FIGURES}

FIG. 1. The radial friction coefficient calculated according to the present model for the approach phase of the ${ }^{64} \mathrm{Zn}(440 \mathrm{MeV})+{ }^{196} \mathrm{Pt}$ reaction as a function of $R$ (solid curve). For comparison the results of the classical model of D.H.E. Gross and H. Kalinowski [32] are shown (dashed curve with stars) and also the results of the microscopic model developed in [15] which are obtained with a constant temperature (dashed curves without stars). The curves correspond to different temperatures which increase from $0.5 \mathrm{MeV}$ (bottom curve) to $2 \mathrm{MeV}$ (upper curve).

FIG. 2. The radial friction coefficients $\gamma_{R R}$ as a function of $R$ for both the approach (solid curve) and departure (dashed curve) stages of the ${ }^{64} \mathrm{Zn}(440 \mathrm{MeV})+{ }^{196} \mathrm{Pt}$ reaction.

FIG. 3. The same as in Fig.2 but for the ${ }^{56} \mathrm{Fe}(480 \mathrm{MeV})+{ }^{208} \mathrm{~Pb}$ reaction.

FIG. 4. Dependence of the total kinetic energy losses calculated by our method (solid curve) and based on the classical model of [32] realized with the code TRAJEC [33] for the ${ }^{64} \operatorname{Zn}(440$ $\mathrm{MeV})+{ }^{196} \mathrm{Pt}$ reaction. The solid and dashed arrows indicate the moment corresponding to the turning point of the trajectory in the present calculations and in the classical model, respectively.

FIG. 5. The same as in Fig.4 but for the ${ }^{56} \mathrm{Fe}(480 \mathrm{MeV})+{ }^{208} \mathrm{~Pb}$ reaction.

FIG. 6. Correction to the nucleus-nucleus interaction potential connected with a rearrangement of the nuclear densities during the approach phase of the reaction for the collision of ${ }^{64} \mathrm{Ni}(320 \mathrm{MeV})$ $+{ }^{208} \mathrm{~Pb}$.

FIG. 7. The nucleus-nucleus interaction potential found in the sudden approximation (dotted line) and with the dynamic correction (dashed line) presented in Fig. 5, and the kinetic energy as a function of the trajectory (solid line) for the ${ }^{64} \mathrm{Ni}(320 \mathrm{MeV})+{ }^{208} \mathrm{~Pb}$ reaction. 


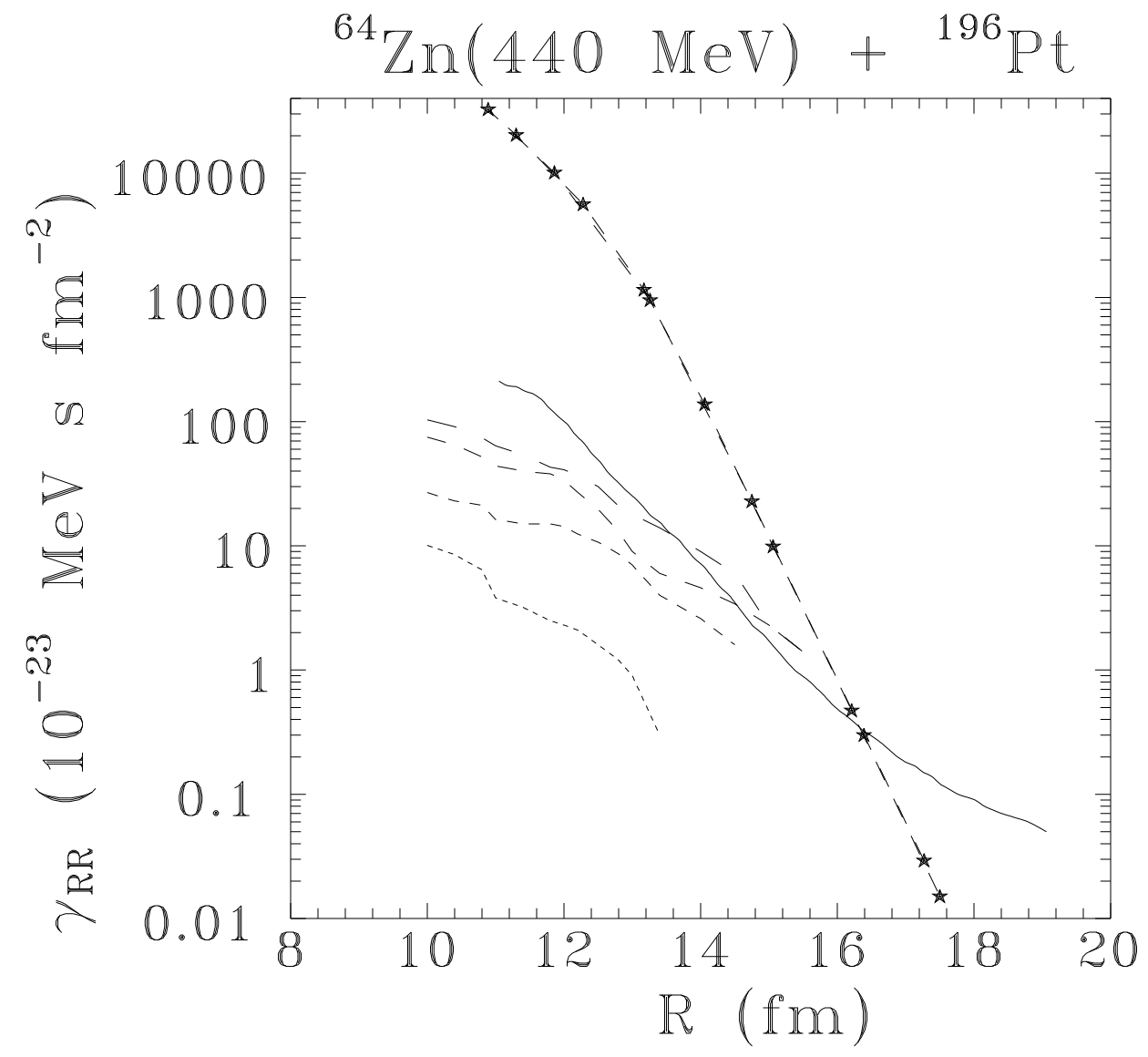

Fig. 1, Adamian G. 


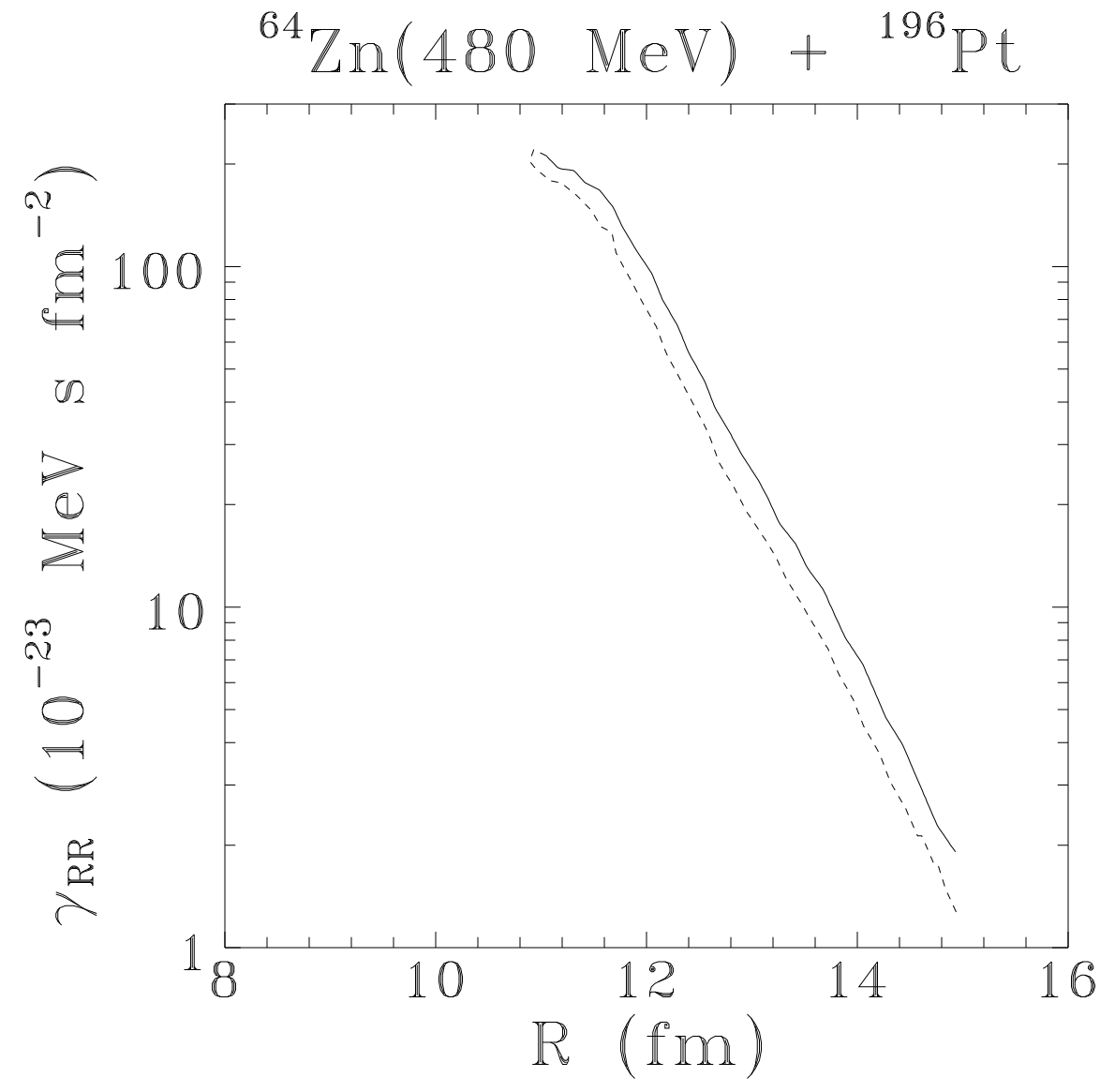

Fig. 2, Adamian G. 


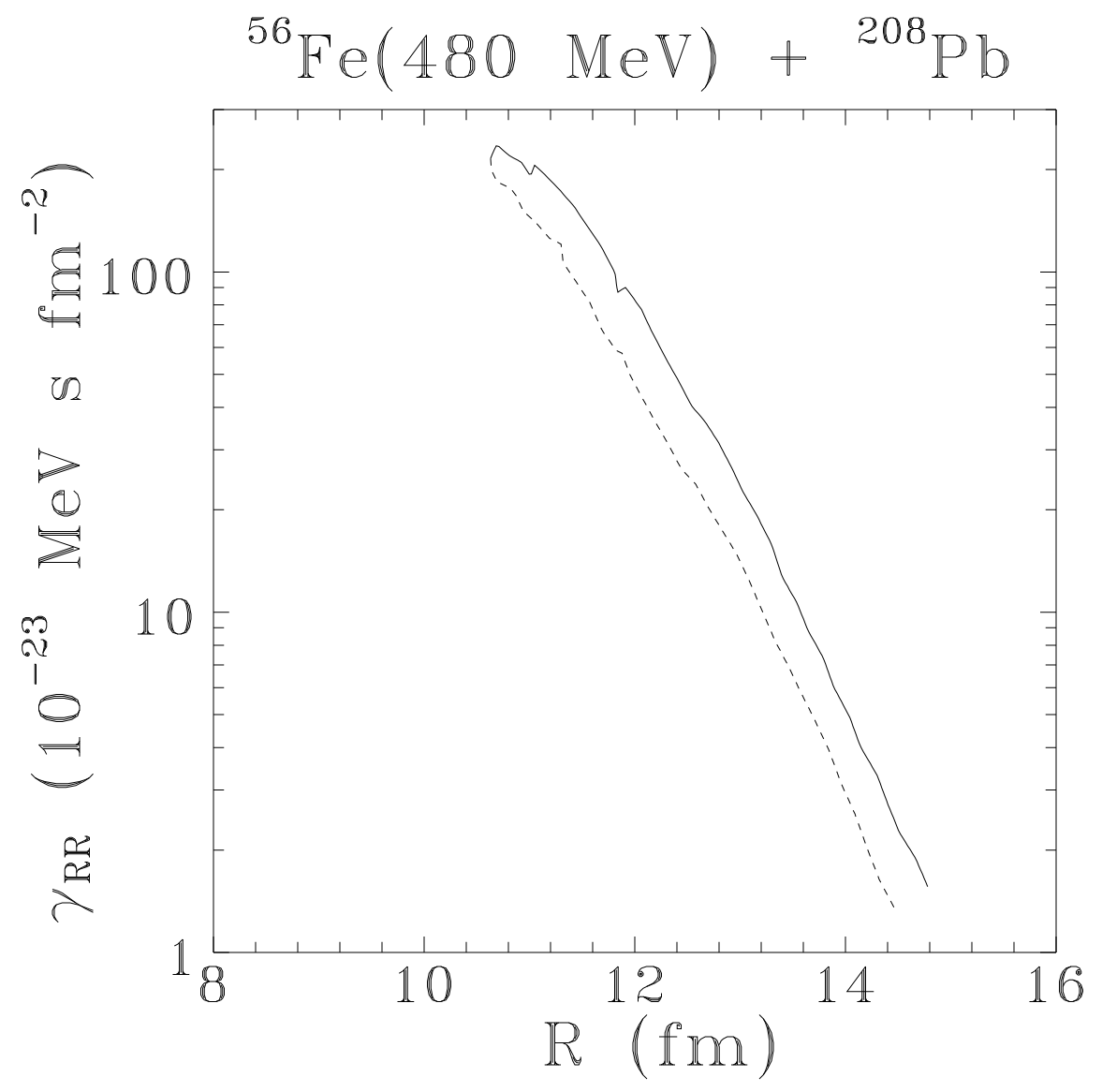

Fig. 3, Adamian G. 


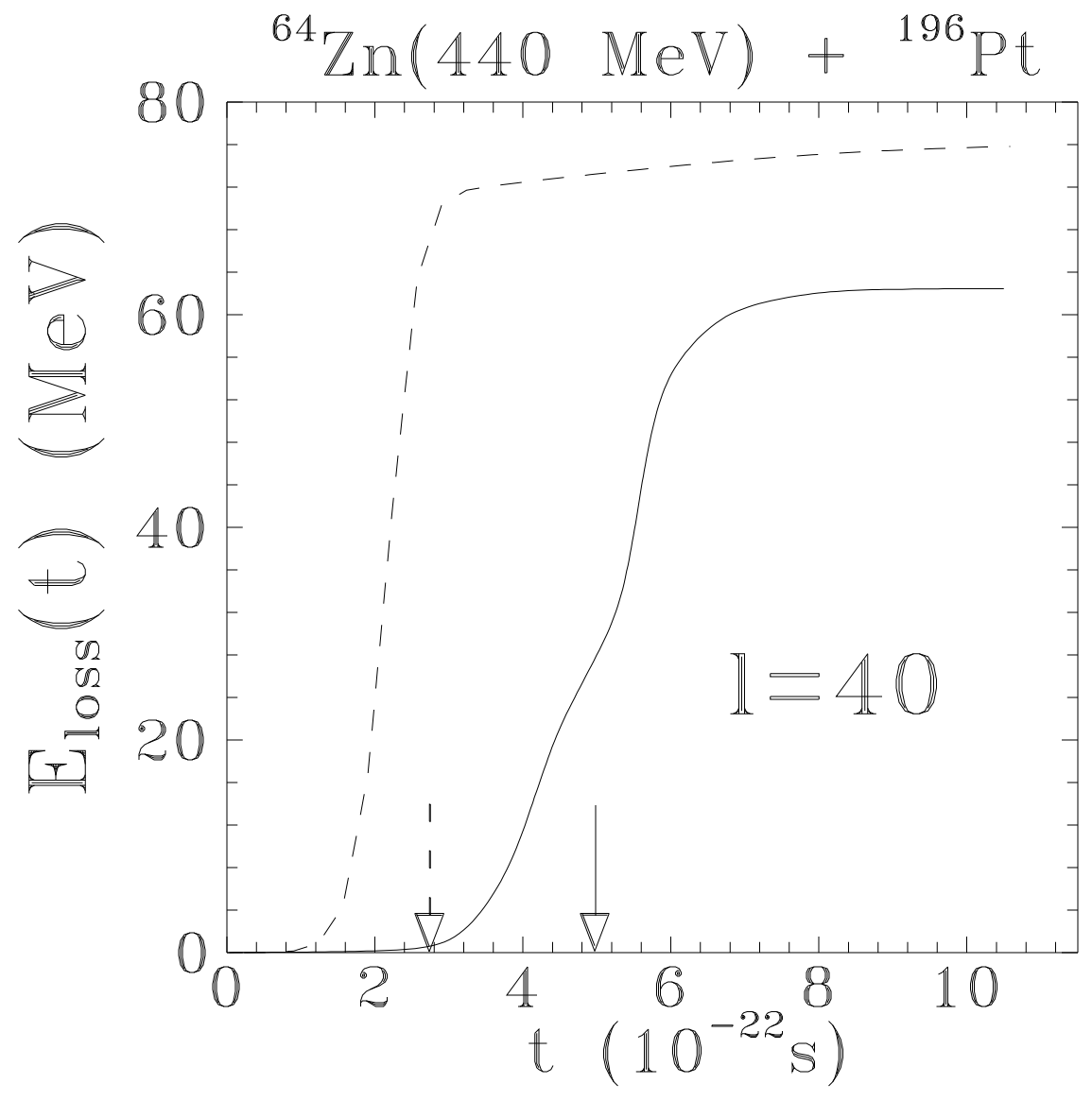

Fig. 4 Adamian G. 


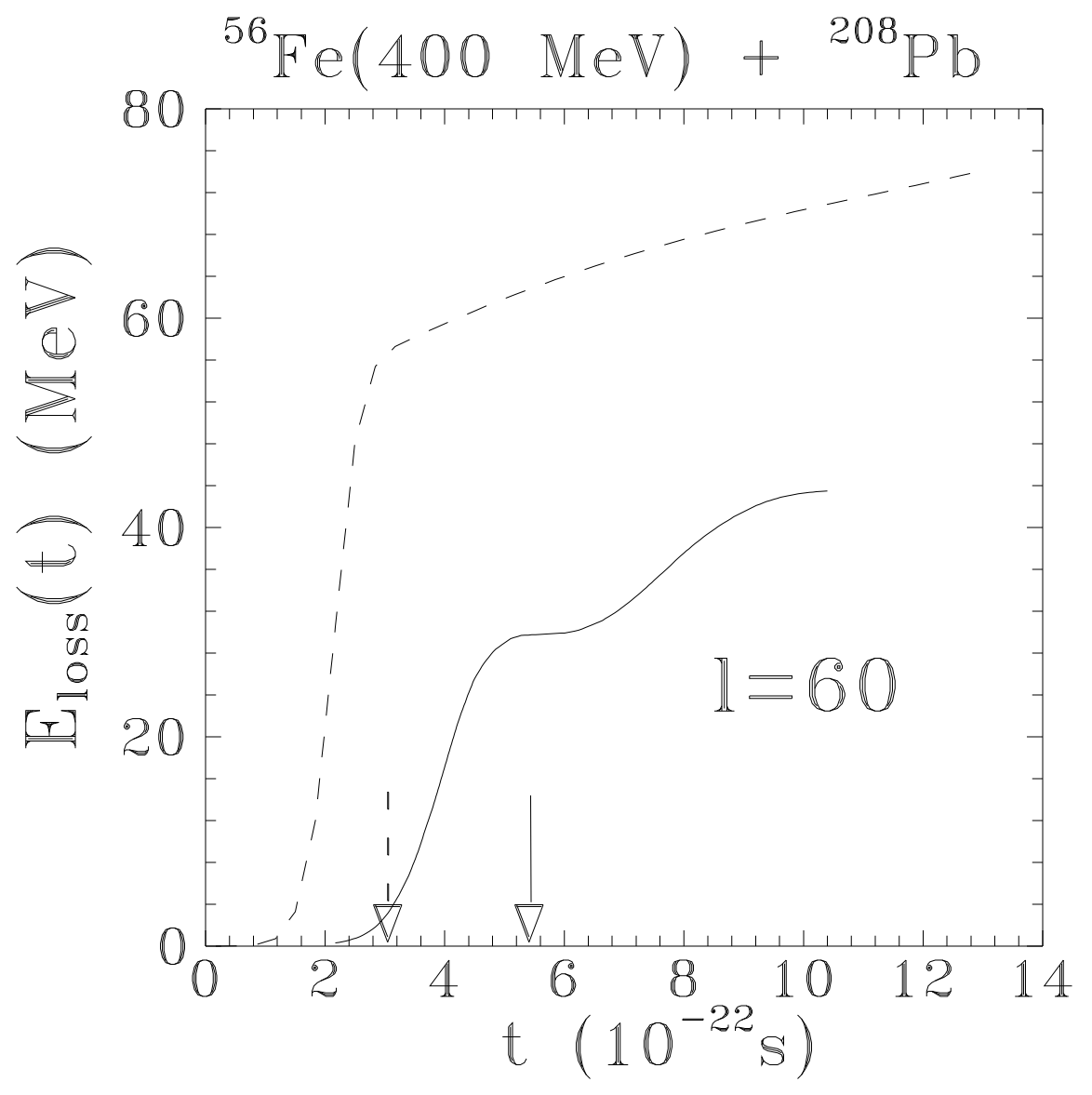

Fig. 5, Adamian G. 


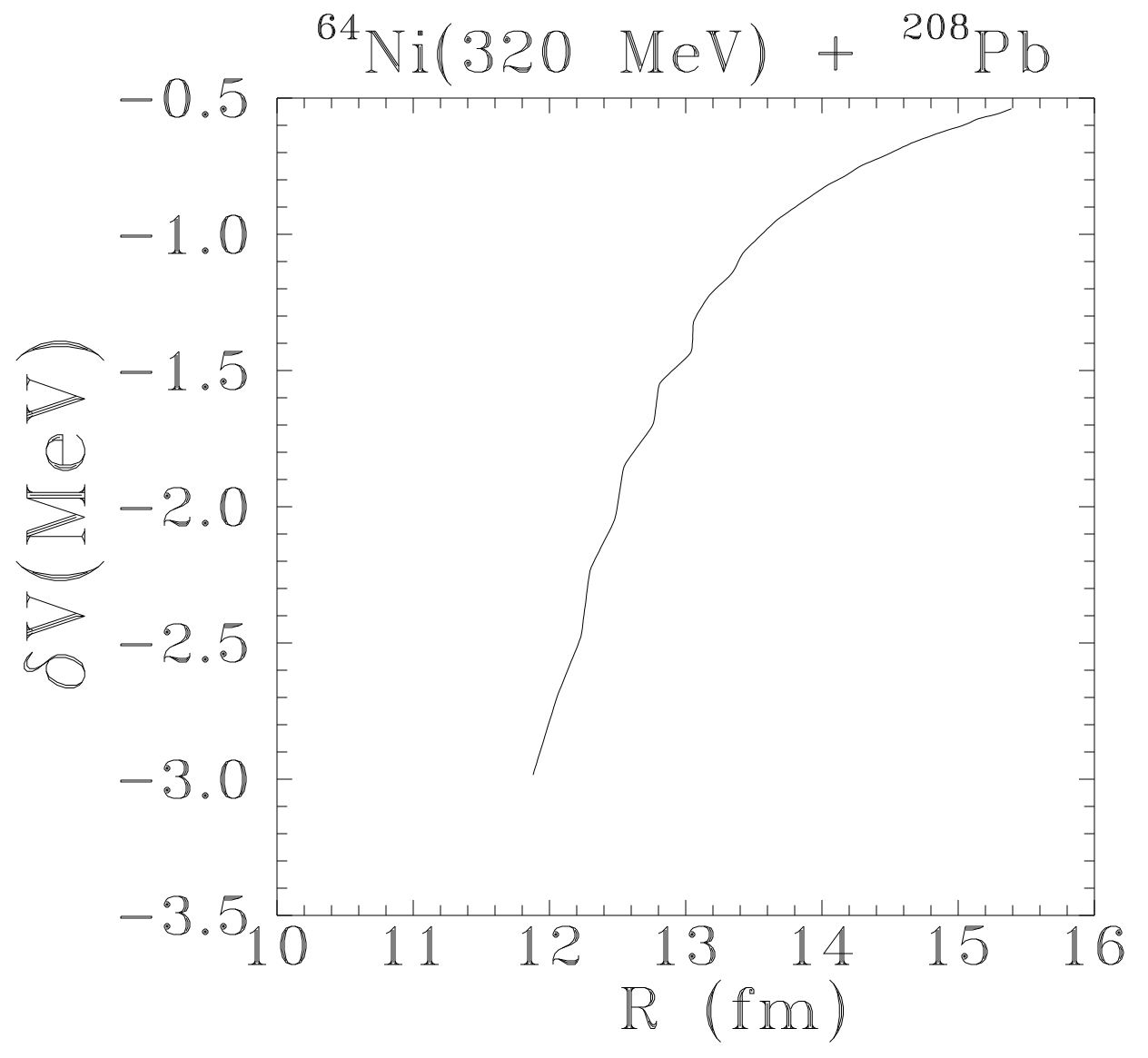

Fig. 6 Adamian G. 


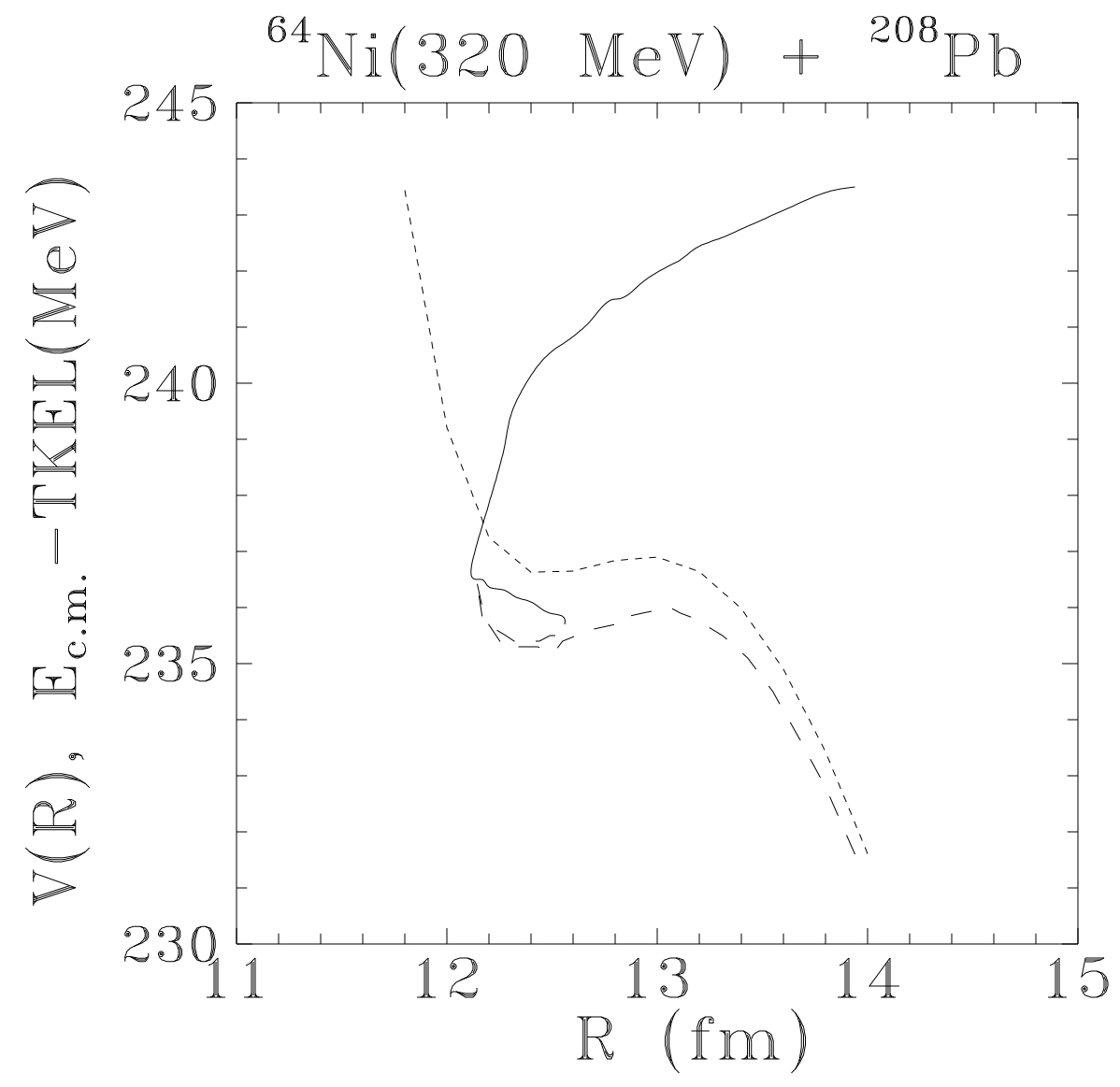

Fig. 7

Adamian G. 